\title{
Undergraduate Research in Mathematics Has Come of Age
}

\author{
Joseph A. Gallian
}

You say you want a revolution

John Lennon

With 2012 being the 25th anniversary of the first NSF REU programs, this is a good time to assess their impact. "Paradigm shift" is a phrase that is frequently overused, but I think it is appropriate to describe a change in the mathematics culture over the past twenty-five years for which REUs have been the catalyst. In 1995 John Greever and I published an article [4] with the title "Challenging a myth: Has undergraduate research in mathematics come of age?" That article concluded with the following paragraph.

Presently, a small but influential group of mathematicians is challenging the myth that undergraduates can't do serious research in mathematics. Our goal is to change the mathematics culture by destroying the myth. When that has been accomplished, we will be able to say with confidence that undergraduate research has indeed come of age."

I think we can now say with confidence that the goal of that group has been achieved.

Joseph A. Gallian is professor of mathematics at the University of Minnesota Duluth. His email address is jga11ian@.d.umn.edu.

Members of the Editorial Board for Doceamus are: David Bressoud, Roger Howe, Karen King, William McCallum, and Mark Saul.

DOI: http://dx.doi.org/10.1090/noti884
Want evidence?

- At the January joint AMS-MAA mathematics meetings in 1991 there were 12 exhibits at the poster session on research by undergraduates. At the same session in 2012 there were 310 posters representing the research of 525 students.

- At the 1993 joint meetings, the first at which the number of undergraduates was tracked, 71 were registered. At the 2012 joint meetings 948 undergraduates were registered.

- In 199515 NSF REU supplements in mathematics were active, ${ }^{1}$ in 2012 89 were active, including ten at the University of Michigan Ann Arbor and five at the University of Minnesota-Twin Cities.

- At the 1996 joint meetings, the first at which the number of talks by were six (three from the same REU). At the 2012 joint meetings, 152 undergraduates gave talks. (Since many

${ }^{1}$ Anyone with an NSF grant is eligible for an REU supplement to support undergraduate students. undergraduates was tracked, there 
of these talks were on work that involved multiple undergraduates, the number of students contributing to these talks is much larger than 152).

- Since 2003 nearly 5,500 undergraduates have given talks on their research at MAA-NSF sponsored regional conferences. Undergraduate attendance at these conferences exceeds 17,000 .

- At an undergraduate research conference in 2009 at Brigham Young University, 95 undergraduates gave talks with 50 of them from BYU.

- Over the past 18 years more than 1,000 mathematicians have taken a four-hour minicourse at the Project NExT summer workshops or the joint mathematics meetings on how to involve undergraduates in research. The 2012 minicourse at the joint mathematics meetings was filled to its capacity of 50 .

- In recent years nearly half of the 125 or so applicants for Project NExT mentioned involving undergraduates in research as one of their career interests.

- Internally funded programs to support research by undergraduates during the academic year and summer at liberal arts colleges, state universities, and Ph.D.-granting institutions are now commonplace. MIT funds summer and academic year research for their undergraduate mathematics students. They also fund a high-level summer research program for high school students with superior talent in mathematics.

- Solo authored papers by undergraduates are being published in leading professional journals: Annals of Mathematics, Annals of Probability, Journal für die reine und angewandte Mathematik (Crelle's Journal), Journal of Algebra, Journal of Combinatorial Theory, Journal of Number Theory, Geometry \& Topology, Journal of Differential Geometry, Transactions of the American Mathematical Society, Proceedings of the American Mathematical Society,
Communications in Algebra, and the SIAM Journal of Discrete Mathematics.

When I became president of the MAA in 2007, I publicly stated that one of my goals was that, by 2015 (the 100th anniversary of the MAA), the number of undergraduates at MAA summer Mathfest would exceed 500 and the number at the joint meetings would exceed 1,000. We are on pace to reach the first goal and the second was too conservative.

The impact of REUs at the joint mathematics meetings is not just being felt at the poster sessions and the short talks. Two of the one-hour invited speakers at the 2011 joint mathematics meetings, Melanie Matchett Wood and Scott Sheffield, got their starts in research at the same REU. The same was true for Manjul Bhargava and Amie Wilkinson at the 2010 joint meetings. In fact, the content of the 2010 invited talk by Bhargava was an extension of the work he had done in an REU in 1995 (see [2] and [3]). Kannan Soundararajan, another invited speaker at the 2011 joint meetings, was a winner of the Morgan Prize for research by an undergraduate. Bhargava gave the three Hedrick lectures at the 2011 MAA Mathfest.

A number of research prizes have gone to REU alumni. Among these are: Manjul Bhargava-the Cole Prize in Number Theory, the Clay Research Prize, the Fermat Prize, and the SASTRA Ramanujan Prize; Davesh Maulik, Daniel Biss, and Manjul Bhargava-the Clay Research Fellowships; Melanie Matchett Wood, Kirsten Graham Wickelgren, Travis Schedler, Jacob Lurie, Mike Develin, Lenhard $\mathrm{Ng}$-American Institute of Mathematics Five-Year Fellowships; Amie Wilkinson-the Satter Prize; and Scott Sheffield - the Loéve International Prize in Probability.

What are the benefits of undergraduate research? Students who engage in research

- gain self confidence,

- improve their writing and speaking skills,

- learn teamwork,

- develop an understanding of what professional mathematicians do,

- receive an introduction to the profession,

- are more likely to enter graduate school ([1] and [5]),

- are more likely to get into a better graduate school, 


\section{CMS Winter Meeting}

Fairmont Queen Elizabeth Hotel

Montreal (Quebec), December 7-10

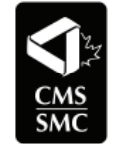

C CENTRE

R DE RECHERCHES

M MATHÉMATIQUES

\section{PUBLIC LECTURES}

Yvar Ekeland (UBC)

Doyle Farmer (Santa Fe Institute)

\section{PLENARY LECTURES}

Graciela Chichilnisky (Columbia)

Martin Nowak (Harvard)

Catherine Sulem (Toronto)

\section{PRIZE LECTURES}

Adrien Pouliot Award to be announced Doctoral Prize - Matthew Kennedy (Carleton) Jeffery-Williams Prize - Roland Speicher

(Univ. des Saarlandes)

\section{SESSIONS}

\section{Special Sessions (MPE2013)}

Celestial Mechanics

Ecological Dynamics under Temporal Variation Epidemiology - Genomics

Epidemiology - Infectious Diseases Geophysics

Operations Research

Probability and Biology

Regular Sessions

Algebraic Combinatorics

Algebraic Number Theory

Analytic Number Theory

Applied Mathematics

Complex Analysis and Operator Theory

Computational Fluid Dynamics

Differential Equations Econometrics

Enumerative Geometry and String Theory

Geometrical Group Theory

Geometry of Algebraic Varieties

History and Philosophy of Mathematics

Mathematical Physics

Mathematics Education Medical Imaging

Operator Theory and Operator Algebras

Probability Theory and Mathematical Physics Quantum Imaging

PDE and Spectral Theory

Symmetries of Differential and Difference Equations

Contributed Papers

AARMS-CMS Graduate Student Poster Session

Scientific Director:

Luc Vinet (Montreal)
- are more likely to do better research in graduate school,

- are more likely to get a Ph.D.,

- are more likely to get a better job offer, and

- are better prepared to do projects required by employers.

Although REUs are showcases for undergraduate research and were the wedge in changing the opinions of many skeptics, they represent only a small fraction of the undergraduates engaged in mathematical research.

What is on the horizon for undergraduate research? More, more, more, and more. More REU-like summer programs, more academic year opportunities for undergraduates to engage in research, more undergraduates attending conferences and presenting, and more research prizes going to people who began doing research as an undergraduate.

It would not surprise me to see an REU alumnus receive a Fields medal before long.

\section{References}

[1] B. B. Alexander, J. A. Foertsch, and S. DAFFinrud, The Spend a Summer with a Scientist Program: An evaluation of program outcomes and the essential elements of success, Madison, WI: University of Wisconsin-Madison, LEAD Center. (1998, July). Report from the Task Force on Women in Science and Engineering, May 2005, page 19 of 65.

[2] M. Bhargava, $P$-orderings and polynomial functions on arbitrary subsets of Dedekind rings. (English summary), J. Reine Angew. Math. 490 (1997), 101-127.

[3] __ On P-orderings, rings of integer-valued polynomials, and ultrametric analysis, J. Amer. Math. Soc. 22 (2009), 963-993.

[4] J. GREEVER and J. GALlian, Challenging a myth: Has undergraduate research come of age?, Council on Undergraduate Research Quarterly, June (1995), 197-198.

[5] B. A. NAgda, S. R. Gregerman, J. Jonides, W. Von HIPPEL, AND J. S. LERNER, Undergraduate studentfaculty research partnerships affect student retention, The Review of Higher Education, 22(1) (1998), 55-72. 